\title{
Contribution of the Neuroanatomy of the Cingulate Gyrus to the Neuroscientific Approach to Depression
}

\author{
Racky Wade-Kane*, El Hadji Makhtar Ba, Momar Camara, Mamadou Habib Thiam \\ Department of Psychiatry and Medical Psychology, Moussa Diop Psychiatric Clinic, Fann National University Hospital Center, \\ Dakar, Senegal \\ Email: ^racky.wade@hotmail.com, bamatar103@yahoo.fr, momarcamara@yahoo.fr, mamadouhabibthiam@gmailcom
}

How to cite this paper: Wade-Kane, R., Ba, El H.M., Camara, M. and Thiam, M.H. (2022) Contribution of the Neuroanatomy of the Cingulate Gyrus to the Neuroscientific Approach to Depression. Open Journal of Psychiatry, 12, 37-48.

https://doi.org/10.4236/ojpsych.2022.121004

Received: November 7, 2021

Accepted: November 29, 2021

Published: December 2, 2021

Copyright (C) 2022 by author(s) and Scientific Research Publishing Inc. This work is licensed under the Creative Commons Attribution International License (CC BY 4.0).

http://creativecommons.org/licenses/by/4.0/

\begin{abstract}
Introduction: Numerous studies show the involvement of the cingulate gyrus in affective disorders, particularly in depression. With a preventive and curative aim, the authors raise questions leading to therapeutic applications such as focal brain stimulation. The cingulate gyrus is the primary target of these brain stimulation therapies for the treatment of depression. The objective of this work is to establish anatomoclinical correlations and to deduce the therapeutic implications. Methodology: Our work is a review of the literature. The inventory of the cingulate gyrus and depression was based on the development of a critical synthesis of bibliographic knowledge. Results: We found a bipartite Brodmann subdivision which evolved into a subdivision into four regions of the cingulate gyrus. Descriptions of the cingulate gyrus boundaries are imprecise and divergent. The anterior end of the anterior cingulate cortex is a confirmed target of stimulation in the treatment of major and resistant depression, thus requiring the authors, a consensus in its delineation. Brodmann's area 25 has been described as the main target of brain stimulation therapies. Dysfunction by local lesion or by alteration of the connectivity of Brodmann's area has repercussions on these different structures to which it is interconnected. These disturbances when they are in the direction of collapse paint a picture similar to major depression. Conclusion: The anterior cingulate cortex is involved in depression. The functional system organization of affectivity will allow new brain stimulation techniques to act on the entire functional system or on one of its components.
\end{abstract}

\section{Keywords}

Cingulate Gyrus, Depression, Anatomy, Psychiatry 


\section{Introduction}

The cingulate gyrus is a part of the limbic lobe located on the medial aspect of the cerebral hemispheres, above the corpus callosum. It corresponds to Brodmann areas $(\mathrm{AB}) 23,24,25,26,29,30,31,32$ and 33 [1]. Many studies have shown the involvement of the cingulate gyrus in affective or mood disturbances [2] [3] [4] [5] [6]. These disturbances in affectivity can point in the direction of collapse or exaggeration.

Depression is a mood disorder or affective disorder [7] characterized by an unpleasant feeling that can reach pain. There are several clinical forms of depression. They all have a common core, which is the depressive syndrome consisting of: depressed mood (sadness, crying, apathy), psychomotor slowing and physical signs [8].

Symptoms of depressive syndrome are complete in severe forms such as major depression, resistant depression and chronic depression. For curative purposes, authors have raised questions which currently lead to therapeutic applications such as focal brain stimulation. The cingulate gyrus is at the forefront of the targets of these brain stimulation therapies including "Deep Brain Stimulation" (DBS) and "Transcranial Magnetic Stimulation" (TMS). The brilliant results obtained reinforce the involvement of the neuro-anatomo-functional dimension in the diagnostic and therapeutic processes of depression, especially in its severe forms. Therefore, the identification of neuroanatomical structures involved in the control of affectivity is of increasing interest day by day in the field of research. Indeed, this interest is noted at several levels. First in the clinic, this update will allow us to understand the mechanism by which the various symptoms of major depression occur. This suggests that practitioners and researchers take into account the biological dimension of mood disorders. Then, this work is also of interest in anatomy in the sense that the development of a recent synthesis on the descriptive and functional aspects of the anatomy of the cingulate gyrus is necessary. This synthesis will complete the first anatomical descriptions of the cingulate gyrus which are certainly existing but rather disparate. She is also contributing to the development of new Atlases of Anatomy of the Central Nervous System. Finally, in imaging, the precise anatomical descriptions produced are of capital interest because they will constitute a descriptive and functional database of this part of the central nervous system. These data will serve as a reference for new image acquisition techniques. This will allow the latter to be close to reality and to keep all their coherence.

Thus, the objective of our work is:

- To establish correlations between the anatomical abnormalities (morphological and functional) of the cingulate gyrus and the clinical aspects occurring during major depression and;

- To deduce the therapeutic implications. 


\section{Methodology}

\subsection{Type of Study}

Our work is a bibliographic study.

\subsection{Materials}

The material used was both physical and electronic works of Anatomy and Psychiatry. The articles were available in the various databases and journals offered by the Jubil entry portal.

We used Excel and World for entering and representing some data in tabular form. The diagram was drawn by hand then scanned and captioned.

The Zotero software allowed the construction of a bibliographic database and the automatic insertion of bibliographic references.

Also, drawing material on paper consisting of colored pencils and felt-tip pens was used to produce a synthesis diagram of the anatomical data.

\subsection{Method}

The review of the literature was based on the development of a critical synthesis of bibliographic knowledge on Cingulate Gyrus and Depression.

We first carried out a well-organized documentary search using the funnel method. This method consists in making a broad then restrictive search.

Through the Jubil entry portal we searched library catalogs and databases according to the type of document sought. The search keywords taken from the title "Cingulate Gyrus and Depression" were translated into Medical Subject Headings (MeSH) terms using the Pubmed database. The same terms were searched directly in Direct Science.

As we went along, we recorded the bibliographic references in Zotero.

From the full texts of the articles that best answered our topic, we assessed the extent, form, and depth of work on the cingulate gyrus and depression. The neuroanatomical data found were synthesized and represented on a diagram produced by freehand.

Also, the analysis of the various consensual aspects and controversies of the subject, led to the formulation of hypotheses that could later be the subject of in-depth research.

\section{Results}

\subsection{Summary of Studies on the Subdivision of the Cingulate Gyrus}

We found a bipartite Brodmann subdivision which evolved into a subdivision into four regions of the cingulate gyrus [Table 1].

\subsection{Synthesis Diagram}

The synthesis diagram shows the limits and subdivision of the cingulate gyrus on a medial view of a left cerebral hemisphere [Figure 1]. 
Table 1. Studies carried out on the subdivision of the cingulate gyrus.

\begin{tabular}{|c|c|c|c|}
\hline TITLE & AUTHORS & \multicolumn{2}{|c|}{ SUBDIVISION OF THE CINGULATE GYRUS } \\
\hline \multicolumn{4}{|c|}{ Brodmann subdivision } \\
\hline \multirow{2}{*}{$\begin{array}{l}\text { Localisation in the cerebral } \\
\text { cortex }\end{array}$} & \multirow{2}{*}{$\begin{array}{l}\text { Brodmann } \\
(1909)\end{array}$} & \multicolumn{2}{|c|}{ Anterior cingulate cortex (ACC): BA24, 25, 32, 33} \\
\hline & & \multicolumn{2}{|c|}{ Posterior cingulate cortex (PCC): BA29, 30, 23, 31} \\
\hline \multirow{2}{*}{$\begin{array}{l}\text { Human cingulate cortex: } \\
\text { surface features, flat maps } \\
\text { and cytoarchitecture }\end{array}$} & \multirow{2}{*}{$\begin{array}{l}\text { Vogt } \\
(1995)\end{array}$} & \multicolumn{2}{|c|}{ Anterior cingulate cortex (ACC): BA24, 25, 32, 33} \\
\hline & & \multicolumn{2}{|c|}{ Posterior cingulate cortex (PCC): BA29, 30, 23, 31} \\
\hline \multicolumn{4}{|c|}{ Vogt review } \\
\hline \multirow{7}{*}{$\begin{array}{l}\text { Pain and emotion } \\
\text { interactions in subregions } \\
\text { of the cingulate gyrus }\end{array}$} & \multirow{7}{*}{$\begin{array}{l}\text { Vogt } \\
(2005)\end{array}$} & & Subgenous anterior cingulate cortex (sACC) \\
\hline & & $\begin{array}{l}\text { Anterior cingulate cortex: } \\
\text { BA24, 25, } 32 \text { and } 33\end{array}$ & $\begin{array}{l}\text { Pre-knotted anterior cingulate cortex } \\
(\mathrm{pACC})\end{array}$ \\
\hline & & \multirow{2}{*}{$\begin{array}{l}\text { Middle cingulate cortex: BA24', } \\
32 \text { ' }\end{array}$} & Anterior middle cingulate cortex (aMCC) \\
\hline & & & Posterior middle cingulate cortex (pMCC) \\
\hline & & \multirow{2}{*}{$\begin{array}{l}\text { Posterior cingulate cortex: } \\
\text { BA23, } 31\end{array}$} & Ventral posterior cingulate cortex (vPCC) \\
\hline & & & Dorsal posterior cingulate cortex (dPCC) \\
\hline & & Retrosplenial cingulate cortex & Part not studied \\
\hline
\end{tabular}

Return of Brodmann subdivision

The Subcallosal Cingulate Gyrus in the Context of Major Depression

Localization of function in anterior cingulate cortex: From psychosurgery to functional

Neuroanatomical correlates of apathy in late-life depression and antidepressant treatment response
Subcallous cingulum:

BA25 + under callused part of BA24 andBA32

(2011) (ACC)

Remaining part of ACC (not studied)

Anterior part: rostral, geneum and anterior

Gasquoine Anterior cingulate cortex

(2013) (ACC)

Posterior part (not studied)

ACC pre-knotted

Yuen Anterior cingulate cortex

(2014) (ACC)

ACC dorsal

\section{Elimination of controversies over MMC by Vogt}

Midcingulate cortex:

Structure, connections,

homologies, functions and

diseases

Anterior MCC

Vogt

(2016)
Middle cingulate cortex (MCC)

Posterior MCC

\section{Discussion}

\subsection{Limits and Constraints}

\subsubsection{Subdivision}

The controversies noted in the literature are partly underpinned by the lack of consensus in the delineation of brain regions. The involvement of Brodmann's 


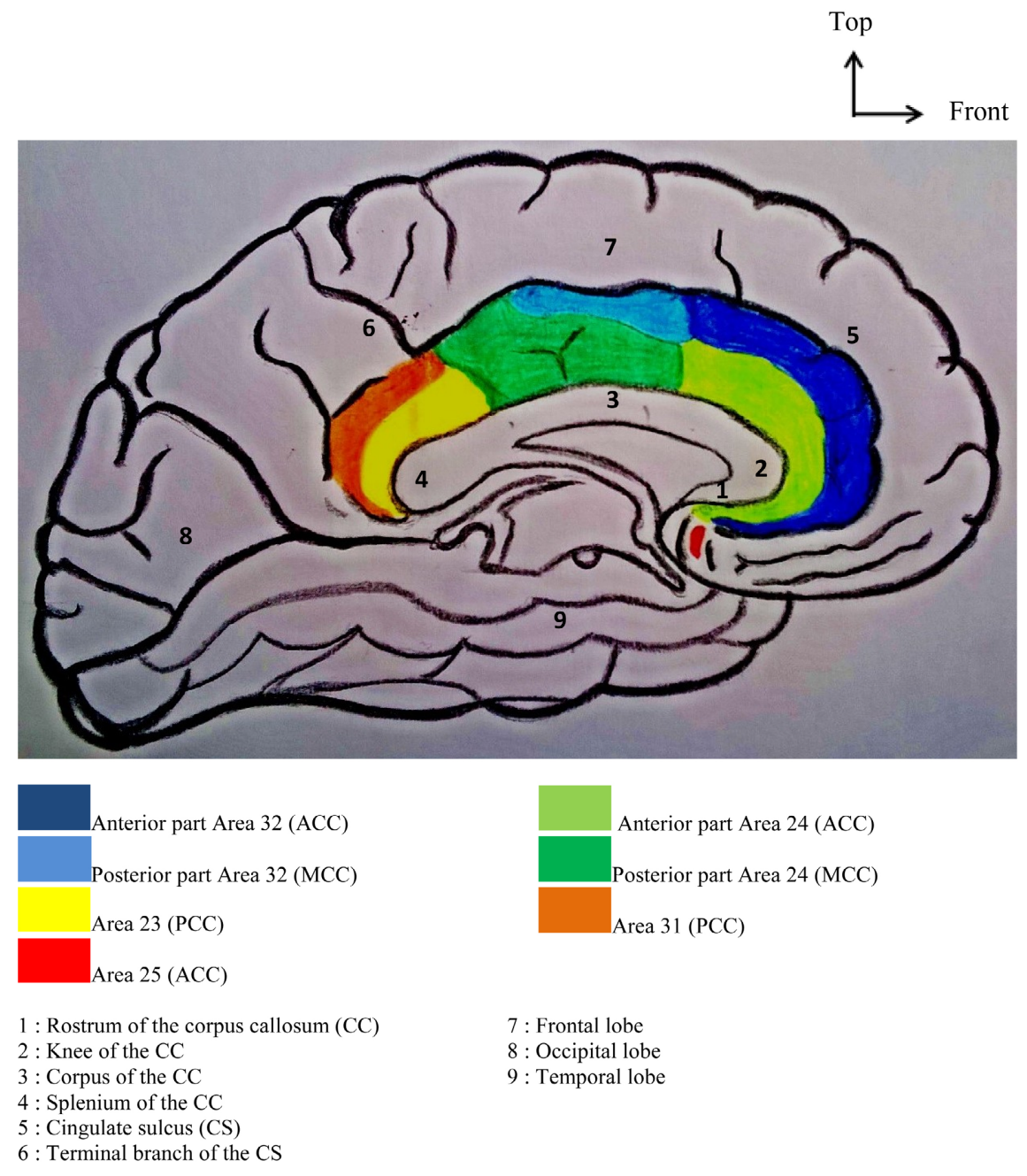

Figure 1. Subdivision of the cingulate gyrus on a medial view of a left cerebral hemisphere.

area 25 in the regulation of affectivity is confirmed by several works [9] [10] [11]. However, some authors consider it to be part of the ACC while others consider it to be part of the ventromedial prefrontal cortex [12]. This leads to divergent conclusions concerning the areas of the brain involved in the affective process.

\subsubsection{Semiological Limits}

Psychiatry is a medical discipline that is not without subtlety when it comes to defining criteria for a given syndrome. The depressive syndrome which is the nucleus of depression is no exception to this criterion. In the literature, patients suffering from major depression are included according to the DSM-IV-TR [9] [13] [14] [15] and DSM-V criteria. On the other hand, those suffering from resistant depression are included according to the criteria of different scales of apathy or depression [9] [10]. Also, the authors used different indicators including apathy and emotion. Tribolet (2000) defines emotion as a usually transient, important variation of the thymic experience. It is a component of affec- 
tivity. It also defines affectivity which corresponds to a thymia refined, orchestrated and often amplified by intelligence and higher psychic functions which constitute the terminal stage of the personality. Affectivity therefore appears as the system which synchronizes both cognitive functions and higher psychic functions such as thought, attention, memory, judgment and mood. The latter is influenced by emotions and instinctual functions [16]. Thus the system organization of the affectivity register comes very close to the new neurobiological approach based on connectivity.

\subsection{Subdivision of the Cingulate Gyrus}

The current subdivision of the cingulate gyrus results from the synthesis of several studies. It went from the simplest to the most complex [1] [17] [18] [19] [20] [21] [22] [23]. Already with Dejerine, the cingulate gyrus was called convolution of the corpus callosum or first convolution of the limbic lobe [18] [24]. It consisted of two parts named according to their situation in relation to the corpus callosum. Brodmann in 1905 proposed on cytoarchitectural bases, a subdivision into two parts called the anterior cingulate cortex (ACC) and posterior cingulate cortex (PCC). This work, admittedly contributory, presented limits concerning the functions of these regions [22]. This is how Vogt in 2005 presented a subdivision of the cingulate gyrus into four parts with definition of their corresponding function. These parts were in addition to ACC and PCC, the middle cingulate cortex (MCC) and retrosplenial cortex (RSC) [6] [21]. The work that followed sparked much controversy, particularly over the existence of the middle cingulate cortex (MCC) which was first described by Vogt. Three authors worked on ACC and they did not consider MCC as a region apart from the cingulate gyrus [5] [9] [13]. They included it in the ACC and matched it to the backbone of the ACC. Which refers to the first subdivision of Brodmann. However, a recent article by Vogt (2016) eliminates any controversy as to whether or not the MCC is considered. First, he specifies that the MCC does not belong to the ACC based on relevant arguments of functional MRI and immunohistochemistry. Then, he describes on precise cyto-architectural bases, the subdivision of the MCC into two parts: the anterior MCC and the posterior MCC. Unlike the MCC, the ACC is far from reaching the same level of consensual subdivision. Currently it is the subject of discord nomenclature with synonymies of cortical areas composing it, without topographic or functional correspondence. This is the case with Brodmann's area 25, which corresponds to both the subgeniculate ACC, the caudal part of the subcallosal cingulum and other inconsistent names. Thus, after a century, the various studies carried out have certainly shown relevant results, but the complexity of the aspects of subdivision and nomenclature of the cingulate gyrus is still relevant today.

\subsection{Synthesis Diagram}

The set of Brodmann areas 24, 25, 31, 32, 33, 23, 26, 29 and 30 constitute the cingulate gyrus. In order to make this diagram comprehensive and simple, these 
areas have been associated with the 4 regions of the cingulate gyrus described by Vogt in 2005. However, this subdivision does not provide information on the internal boundaries of the 4 regions of the cingulate gyrus. Nevertheless, we found in the literature anatomical landmarks [19] allowing us to represent the anterior and posterior limits of the MCC in our diagram. The MCC corresponds to the middle third of the corpus callosum and its posterior limit is located just before the reflection of the marginal branch of the cingulate sulcus. Thus, on this medial view of a left cerebral hemisphere, area 25 and the rostral part of areas 24 , 32,33 correspond to ACC, the caudal part of areas 24, 32 and 33 represents MCC, areas 23 and 31 correspond to the PCC and finally the areas 26, 29 and 30 constitute the RSC. Area 33 and splenial areas 26, 29 and 30 are not visible in our diagram because they are located deep in the furrow of the corpus callosum. Area 33 is buried in the sulcus of the corpus callosum as a narrow strip of cortex near the knee of the corpus callosum. The areas of the RSC are located on the anterior surface of the PCC facing the splenium and within the sulcus of the corpus callosum. The descriptions given on the limits of the cingulate gyrus are imprecise and above all divergent. From the work of Dejerine (1895) and Brodmann (1905), to those of other more recent authors, this imprecision persists, which mainly concerns the anterior and posterior extremities of the cingulate gyrus. For some authors, the posterior end corresponds to the splenial region [21] [22]. However, in Drake's work, the limbic lobe isthmus is considered to be the terminal or posterior end of the cingulate gyrus. It is a narrow strip of cortex separating the cingulate gyrus from the parahippocampal gyrus. The same is true for the anterior extremity which corresponds to Brodmann's area 25. Its delimitation and denomination are the subject of controversy in the literature. It is sometimes attached to the knee of the corpus callosum [18], sometimes to the caudal part of the rostrum [21] [22]. The synthesis of the various neuroanatomical descriptions allowed us to specify its limits on the diagram. Brodmann's area 25 is bounded in front and below by Brodmann's area 11, behind the paraterminal gyrus from which it is separated by the posterior para-olfactory sulcus. Above and in front it is limited by the cranial part of the subcallous gyrus and a short vertical groove called the anterior para-olfactory groove. However, for some authors, it belongs to the prefrontal cortex. This partly explains the divergent conclusions found in the literature. The proponents for the membership of Brodmann's area 25 to the pre-frontal cortex used a methodology based on imaging (PET, CT, MRI) and the proponents for the membership of this Brodmann area 25 to the cingulate cortex have used in addition to imagery arguably more precise cyto-architectonics.

Moreover, this anterior extremity (Brodmann's area 25) is arousing much interest with new focal brain stimulation therapies applied to neurological and psychiatric pathologies [10] [25] [26] [27]. It constitutes a confirmed target of stimulation in the treatment of major and resistant depression.

Faced with these innovative therapeutic perspectives and the disagreements described above, it is necessary to carry out work whose main objective would be 
to find a consensus in the delimitation and nomenclature of this region. Also, this would make it possible to make the data from the literature that already exist better usable. Part of our work is a start.

\subsection{Cingulate Gyrus and Depression}

The first experimental studies in animals linked emotional behavior to the cingulate gyrus. Lesions of the cingulate gyrus in monkeys have led to the disappearance of their aggressiveness, an exaggerated taming and a disinterest in the face of danger [28] [29]. These animal experiments paved the way for cingulectomy as a psychosurgical method. Patients with mental disorders whose depression improved rapidly after cingulectomy. Also, thanks to neuroimaging, abnormalities in brain activity of ACC were found in patients suffering from depression [13]. From then on, researchers were interested in the identification of neuroanatomical structures involved in the control of affectivity. The first results showed that only the anterior part of the ACC is involved in the emotion, the posterior part being activated during pain responses. The second results obtained incriminated the subcallous gyrus of ACC and more precisely Brodmann's area [5] [9] as the control center of the affectivity system. In fact, abnormalities in the brain activity of Brodmann's area have been found in depressive patients. Also in a stimulation experiment in humans (thoughts of sad events) the results showed significant brain activity at Brodmann's area.

Functionally, this area is interconnected with other regions of the cingulate cortex such as the posterior ACC, MCC and PCC. It is also related to the ventro-medial prefrontal cortex, the dorsolateral prefrontal cortex, the hypothalamus, the supplementary motor area, the reticulate formation, the amygdala, the hippocampus and the nucleus accumbens [1] [5] [11] [29] [30]. Consequently, dysfunction by local lesion or by alteration of the connectivity of Brodmann's area will have repercussions on these different structures to which it is interconnected. These may be disturbances of interest to:

- Cognitive functions such as attention, thinking (dorsolateral prefrontal cortex), memory (hippocampus);

- Vegetative functions, appetite and libido (hypothalamus);

- Sleep (reticulate formation);

- Interest and motivation (nucleus acumbens);

- Pre-motor functions (additional motor area).

These disturbances when they go in the direction of a collapse give a picture similar to the major depression characterized by:

- Depressed mood with sadness, listlessness, anhedonia (loss of pleasure) and lack of interest;

- Psychomotor slowing down with bradypsychia (slowing down of thought), rare movements which may be due to a loss of movement initiation;

- Physical signs: Ey (1989) in his book describes digestive signs such as anorexia, nausea, saburral state of the digestive tract, constipation or diarrheal 
debacles. Cardiovascular examination shows disturbances in pulse and blood pressure with hypotension in the stuporous form and hypertension in the anxious form. Amenorrhea is common. The neuro-vegetative signs lead to vagotonic syndrome in the stuporous form and suggest a stressful adrenergic reaction in the anxious form [8].

- Amnesia;

- Hypoprosexia (decreased attention);

- A decrease in libido;

- Insomnia.

These anatomo-clinical correlations justify the application of new therapeutic approaches to focal brain stimulation. "Deep Brain Stimulation" (DBS) and "Transcranial Magnetic Stimulation" (TMS) have been shown to be effective in the treatment of major depression and resistant depression [10] [31]. The focal stimulation of the primary center of the emotion will propagate to the levels of the other structures to which it is interconnected [5]. Thus it will raise their level of activity and give a state of basal emotional tone. This stimulation can also concern the white matter fibers which connect these different structures.

In addition, other authors inspired by the work of Papez, organize the representation of emotion at the level of the brain, into two components [32]:

- A physical component connected to the hypothalamus and which underlies the physical, somatic and vegetative signs, during depression and

- A psychic component involved in "conscious perception" and provided by the cingulate cortex.

\section{Recommendations}

This work carried out on depression and the cingulate gyrus suggests that we push our reflection on the following points:

- The precise delimitation of a psychic cortical area and the knowledge of the trajectory of the fibers which connects it to other structures, will make it possible to act on the entire functional system or on one of its components. Thus, we recommend focal stimulation on white matter fibers to act on a dominant component of depression. This is an example of a clinical form of anxiety depression. The stimulation will target the white fibers originating from the cingulate cortical area and which are in connection with the structures that govern anxiety. On the other hand for a complete depressive syndrome in a context of major, resistant or chronic depression, we indicate a cortical stimulation which will diffuse at the level of the other interconnected structures thanks to the fibrous projections;

- The integration of anatomical data obtained in neuro-navigation systems; which will help in pinpointing the exact site during brain stimulation;

- The establishment of a mapping of psychic brain areas which also takes into account the connectivity between the different brain structures;

- The revision of the anatomical nomenclature of the different regions of the 
cingulate gyrus in order to eliminate the many synonymies without topographical or functional correspondence. This revision will also make the much research work on the cingulate gyrus and depression more useful.

\section{Conclusion}

Emotion is a primitive instance that humans share with other mammals. Affection involves highly differentiated structures including the prefrontal cortex. Its system organization responds to the new neurobiological approach based on the connectivity of neuro-anatomical structures. Brodmann's area of the anterior cingulate cortex (ACC) is implicated in depression despite the complexity of the neuroanatomical and psychiatric aspects noted in the various studies. This complexity opens the way for fundamental, clinical and synthetic studies.

\section{Conflicts of Interest}

The authors have no conflict of interest.

\section{References}

[1] FitzGerald, M.J.T. and Folan-Curran, J. (2003) Systèmes olfactif et limbique. In: Neuro-anatomie clinique et neurosciences connexes, 4th Edition, Maloine, Paris, 275-292.

[2] Burgdorf, J. and Panksepp, J. (2006) The Neurobiology of Positive Emotions. Neuroscience \& Biobehavioral Reviews, 30, 173-187. https://doi.org/10.1016/j.neubiorev.2005.06.001

[3] Em-premium (2016) Anomalies cérébrales structurales et fonctionnelles dans le trouble bipolaire. https://www.em-consulte.com/article/905232/anomalies-cerebrales-structurales-et-f onctionnelle

[4] Vogt, B.A. (2014) Submodalities of Emotion in the Context of Cingulate Subregion. Cortex, 59, 197-202. https://doi.org/10.1016/j.cortex.2014.04.002

[5] Hamani, C., Mayberg, H., Stone, S., Laxton, A., Haber, S. and Lozano, A.M. (2011) The Subcallosal Cingulate Gyrus in the Context of Major Depression. Biological Psychiatry, 69, 301-308. https://doi.org/10.1016/j.biopsych.2010.09.034

[6] Vogt, B.A. (2005) Pain and Emotion Interactions in Subregions of the Cingulate Gyrus. Nature Reviews Neuroscience, 6, 533-544. https://doi.org/10.1038/nrn1704

[7] OMS G (1993) Troubles mentaux et troubles du comportement: Descriptions cliniques et directives pour le diagnoctic. In: Classification Internationale de Maladies Dixième révision (CIM10), 10th Edition, Masson, Paris, 10.

[8] Ey, H. (1989) Manuel De Psychiatrie. 6th Edition, Masson, Paris, 597. https://doi.org/10.1016/B978-2-294-71158-9.50030-8

[9] Yuen, G.S., Gunning, F.M., Woods, E., Klimstra, S.A., Hoptman, M.J. and Alexopoulos, G.S. (2014) Neuroanatomical Correlates of Apathy in Late-Life Depression and Antidepressant Treatment Response. Journal of Affective Disorders, 166, 179-186. https://doi.org/10.1016/j.jad.2014.05.008

[10] Taghva, A.S., Malone, D.A. and Rezai, A.R. (2013) Deep Brain Stimulation for Treatment-Resistant Depression. World Neurosurgery, 80, S27.e17-24. https://doi.org/10.1016/j.wneu.2012.10.068 
[11] Fales, C.L., Barch, D.M., Rundle, M.M., Mintun, M.A., Snyder, A.Z., Cohen, J.D., et al. (2008) Altered Emotional Interference Processing in Affective and Cognitive-Control Brain Circuitry in Major Depression. Biological Psychiatry, 63, 377-384. https://doi.org/10.1016/j.biopsych.2007.06.012

[12] Finger, E.C., Marsh, A.A., Mitchell, D.G., Reid, M.E., Sims, C., Budhani, S., et al. (2008) Abnormal Ventromedial Prefrontal Cortex Function in Children with Psychopathic Traits during Reversal Learning. Archives of General Psychiatry, 65, 586-594. https://doi.org/10.1001/archpsyc.65.5.586

[13] Gasquoine, P.G. (2013) Localization of Function in Anterior Cingulate Cortex: From Psychosurgery to Functional Neuroimaging. Neuroscience \& Biobehavioral Reviews, 37, 340-348. https://doi.org/10.1016/j.neubiorev.2013.01.002

[14] Holtzheimer, P.E., Kelley, M.E., Gross, R.E., Filkowski, M.M., Garlow, S.J., Barrocas, A., et al. (2012) Subcallosal Cingulate Deep Brain Stimulation for Treatment-Resistant Unipolar and Bipolar Depression. Archives of General Psychiatry, 69, 150-158. https://doi.org/10.1001/archgenpsychiatry.2011.1456

[15] Coryell, W., Nopoulos, P., Drevets, W., Wilson, T. and Andreasen, N.C. (2005) Subgenual Prefrontal Cortex Volumes in Major Depressive Disorder and Schizophrenia: Diagnostic Specificity and Prognostic Implications. American Journal of Psychiatry, 162, 1706-1712. https://doi.org/10.1176/appi.ajp.162.9.1706

[16] Tribolet, S. and Shahidi, M. (2000) Précis de sémiologie des troubles psychiques. Editions Heures de France, Paris, 456.

[17] Wikipédia (2016) Aires de Brodmann. https://fr.wikipedia.org/wiki/Aires de Brodmann\#cite ref-1

[18] Dejerine, J., Dejerine-Klumpke, A. (1895) Anatomie des centres nerveux. Tome 1. Rueff et Cie, Paris, 828. https://gallica.bnf.fr/ark:/12148/bpt6k76498h/f19.item

[19] Vogt, B.A. and Palomero-Gallagher, N. (2012) Cingulate cortex. In: Mai, J. and Paxinos, G., Eds., The Human Nervous System, 3rd Edition. Elsevier Inc., Amsterdam, 943-987.

https://cingulumneurosciences.net/wp-content/uploads/2021/08/9.-Vogt-Human-C ingulate-Cortex.pdf

[20] Bouriez, D. (2012) Évolution des connaissances sur le cingulum de Déjerine à nos jours et reconstitution tridimensionnelle à partir d'IRM de quatre étudiants volontaires. Morphologie, 96, 110. https://doi.org/10.1016/j.morpho.2012.08.105

[21] Vogt, B.A., Nimchinsky, E.A., Vogt, L.J. and Hof, P.R. (1995) Human Cingulate Cortex: Surface Features, Flat Maps, and Cytoarchitecture. Journal of Comparative Neurology, 359, 490-506. https://doi.org/10.1002/cne.903590310

[22] Brodmann, K. (2005) Localisation in the Cerebral Cortex [Vergleichende Lokalisationslehre der Grosshirnrinde in ihren Prinzipien Dargestellt auf Grund des Zellenbaues]. Imperial College Press, London.

[23] Vogt, B.A. (2016) Midcingulate Cortex: Structure, Connections, Homologies, Functions and Diseases. Journal of Chemical Neuroanatomy, 74, 28-46. https://doi.org/10.1016/j.jchemneu.2016.01.010

[24] Rouvière, H. and Delmas, A. (2002) Anatomie descriptive du système nerveux central. In: Système nerveux central, voies et centres nerveux, 15th Edition, Masson, Paris, 84-85.

[25] Merkl, A., Schneider, G.-H., Schönecker, T., Aust, S., Kühl, K.-P., Kupsch, A., et al. (2013) Antidepressant Effects after Short-Term and Chronic Stimulation of the Subgenual Cingulate Gyrus in Treatment-Resistant Depression. Experimental Neurology, 249, 160-168. https://doi.org/10.1016/j.expneurol.2013.08.017 
[26] Fox, M.D., Buckner, R.L., White, M.P., Greicius, M.D. and Pascual-Leone, A. (2012) Efficacy of Transcranial Magnetic Stimulation Targets for Depression Is Related to Intrinsic Functional Connectivity with the Subgenual Cingulate. Biological Psychiatry, 72, 595-603. https://doi.org/10.1016/j.biopsych.2012.04.028

[27] Lozano, A.M., Mayberg, H.S., Giacobbe, P., Hamani, C., Craddock, R.C. and Kennedy, S.H. (2008) Subcallosal Cingulate Gyrus Deep Brain Stimulation for Treatment-Resistant Depression. Biological Psychiatry, 64, 461-467.

https://doi.org/10.1016/j.biopsych.2008.05.034

[28] Glees, P., Cole, J., Whitty, C.W.M. and Cairns, H. (1950) The Effects of Lesions in the Cingular Gyrus and Adjacent Areas in Monkeys. Journal of Neurology, Neurosurgery and Psychiatry, 13, 178-190. https://doi.org/10.1136/jnnp.13.3.178

[29] Vitte, E. and Chevallier, J.-M. (1998) Le cerveau. In: [4] Neuro-Anatomie, Flammarion, Paris, 143

[30] Rothé, M. (2010) Activités spécifiques du cortex cingulaire antérieur et du cortex préfrontal dorsolatéral et interactions lors de l'adaptation des comportements. Université Claude Bernard-Lyon I, Lyon. https://tel.archives-ouvertes.fr/tel-00710536

[31] Lefaucheur, J.-P., André-Obadia, N., Poulet, E., Devanne, H., Haffen, E., Londero, A., et al. (2011) Recommandations françaises sur l'utilisation de la stimulation magnétique transcrânienne répétitive (rTMS): Règles de sécurité et indications thérapeutiques. Neurophysiologie Clinique, 41, 221-295.

https://doi.org/10.1016/j.neucli.2011.10.062

[32] Berry, M., Bannister, L.H. and Standring, S.M. (1995) Nervous System. In: Gray’s Anatomy, 38th Edition, Churchill Livingstone, New York, 1106-1115. 